\title{
Complete Remission of Pancreatic Pseudoaneurysm Rupture with Arterial Embolization in a Patient with Poor Risk for Surgery: A Case Report
}

\author{
Kuang-En $\mathrm{Chu}^{\mathrm{a}}$ Cheuk-Kay Sun ${ }^{\mathrm{a}}$ Chin-Chu Wu ${ }^{\mathrm{b}}$ \\ Kuo-Ching Yang ${ }^{\mathrm{a}}$ \\ ${ }^{a}$ Division of Gastroenterology, Department of Internal Medicine, and \\ ${ }^{b}$ Department of Radiology, Shin Kong Wu Ho-Su Memorial Hospital, Taipei, \\ Taiwan
}

\section{Key Words}

Pseudoaneurysm · Pancreatitis · Angiography · Arterial embolization

\begin{abstract}
Pancreatic pseudoaneurysm is a rare vascular complication of chronic pancreatitis resulting from erosion of the pancreatic or peripancreatic artery into a pseudocyst that is identified as a pulsating vascular malformation which may lead to lethal complications if left untreated. Many publications in the literature consider angiography as the first step in the management of pancreatic pseudoaneurysm to stabilize the patient's critical condition; it should be followed by surgical intervention as the definite treatment. We report a rare case of pancreatic pseudoaneurysm rupture with hemodynamic embarrassment in a critical patient with multiple comorbid conditions and poor risk for surgery who responded dramatically to angiographic management as a single therapeutic modality without further surgical intervention. The results observed in our patient suggest that pancreatic pseudoaneurysm may be successfully managed with angiography only and that not all cases require surgical intervention. This is particularly relevant in critically ill patients in whom surgical intervention would be unfeasible.
\end{abstract}

\section{Introduction}

Pancreatic pseudoaneurysm is the result of erosion of the pancreatic or peripancreatic artery into a pseudocystic formation; as a consequence a communication between the vasculature and the pseudocyst is formed. The most commonly involved arteries are the splenic artery in up to $50 \%$ of cases, and less 
frequently the gastroduodenal artery, the pancreaticoduodenal artery, the superior mesenteric artery, the left gastric artery and the hepatic artery. A pseudoaneurysm is different from a true aneurysm in the sense that the wall of the pseudoaneurysm is composed of fibrous tissue instead of a well-formed arterial wall, and one of the characteristics is its rapid growth as a pulsating vascular malformation [1]. Pancreatic pseudoaneurysm may also develop as an abnormal aneurysmal dilatation in the absence of a pseudocyst due to digestion of the vasculature walls by the pancreatic enzymes. It is a rare vascular complication of chronic pancreatitis with an estimated prevalence of $<10 \%$ [2]. Though these vascular complications may follow a unpredictable evolution, they can precipitate lethal complications such as rupture and massive bleeding, with a mortality rate as high as $12.5 \%$ even in treated patients and $>90 \%$ if patients are left untreated $[3,4]$.

Angiography is the standard tool for the diagnosis of pancreatic pseudoaneurysm, and treatment planning with enhanced CT scans has a sensitivity and specificity up to 95 and $90 \%$, respectively [5]. Although there is a consensus that all these vascular malformations should be treated, the current major controversy is whether transarterial catheter embolization should be the definite management or whether it should be only a palliative approach followed by surgical intervention [6]. We herein report a case of sudden pancreatic pseudoaneurysm rupture in a patient unsuitable for surgical intervention who responded successfully and dramatically to transarterial catheter embolization as a single therapeutic management.

\section{Case Report}

A 56-year-old man with a medical record of recent brain infarction under antiplatelet therapy with clopidogrel, chronic renal failure, arterial hypertension and long-standing alcoholic habit presented to the emergency room for progressive dyspnea, fever, diaphoresis, moderate to intense pain in the epigastrium radiating to the back, anorexia and intermittent melena in the past 2 weeks. The pain had not decreased by the usual analgesics and had even worsened in the last 3 days. The patient used to drink at least $40 \mathrm{~g}$ of alcohol daily for many years. Physical examination revealed a cachectic, pale, acutely ill, dyspneic, oriented and febrile $\left(38^{\circ} \mathrm{C}\right)$ patient, with normal heart sounds $(97 \mathrm{bpm})$ and arterial pressure $(144 / 86 \mathrm{~mm} \mathrm{Hg})$. The abdomen was prominently tender in the left upper epigastric area and the tenderness was increased with palpation. Chest auscultation showed diffuse bilateral rhonchi and wheezing and markedly decreased breathing sounds in the right lung. Rectal examination showed melena. Laboratory tests revealed the following data: leukocytosis $\left(12,000 / \mathrm{mm}^{3}\right)$, low hemoglobin level $(8.6 \mathrm{~g} / \mathrm{dl})$, normal platelet count $\left(39.2 \times 10^{4} / \mu \mathrm{l}\right)$, elevated serum creatinine $(5.9 \mathrm{mg} / \mathrm{dl})$, lipase $(2,125 \mathrm{IU} / \mathrm{l})$, amylase (771 IU/l), LDH (585 IU/l) and glucose (141 mg/dl), and normal aspartate aminotransferase (35 IU/l) and total bilirubin levels $(0.3 \mathrm{mg} / \mathrm{dl})$. A chest film disclosed bilateral pneumonia with a large amount of right parapneumonic pleural effusion; thoracocentesis was undertaken in order to drain out the pleural effusion. Computed tomography showed an atrophic pancreas and four variable-sized cystic lesions in the pancreatic body and tail, the largest of them, located in the body, measuring $3.97 \mathrm{~cm}$. Calculi in the gallbladder with no overt biliary dilatation were noted as well.

The patient was hospitalized with a diagnosis of chronic pancreatitis with pseudocyst formation, pneumonia with parapneumonic pleural effusion, septicemia, chronic renal failure and recent brain stroke. Four days after hospitalization, a follow-up transabdominal ultrasound revealed a rapidly enlarging pseudocyst in the tail of the pancreas which reached $10 \mathrm{~cm}$ in size. Endoscopic retrograde cholangiopancreatography was undertaken. Multiple actively bleeding duodenal ulcers were noted. Extravasation of the contrast medium over the body portion of the main pancreatic duct was demonstrated and a communication between the pseudocysts and the main pancreatic duct was suspected. A $5 \mathrm{Fr}, 12 \mathrm{~cm}$-long Zimmon pancreatic stent was inserted into the main pancreatic duct after endoscopic sphincterotomy. The patient was placed on treatment with proton pump inhibitors 
for the duodenal ulcer bleeding. He showed a relatively acceptable clinical course with regression of the pancreatic pseudocysts in two subsequent transabdominal ultrasound follow-ups. On the 25th day of hospitalization, the patient presented a sudden, sharp left hemiabdominal pain associated with general pallor, diaphoresis, tachycardia and abdominal distension, and systolic arterial pressure dropped to $70 \mathrm{~mm} \mathrm{Hg}$. The pseudocyst located in the pancreatic tail which decreased in size after main pancreatic duct stenting showed turbulent blood flow as visualized by color Doppler imaging (Toshiba Xario SSA-660A), establishing the tentative diagnosis of pancreatic pseudoaneurysm with probable rupture according the patient's clinical settings. Emergent contrast-enhanced computed tomography of the abdomen was undertaken and showed hyperdense hemorrhagic content in the pseudocyst (fig. 1). Sudden rupture of a pancreatic pseudoaneurysm with active bleeding was highly suspected, and surgeons were immediately called to evaluate the possibility of surgical hemostasis in this poor-risk and rapidly deteriorating patient with hemodynamic embarrassment. However, the surgeons considered that surgical intervention would not be advisable in this shocked patient with multiple severe systemic illness and very poor general performance. An emergent celiac angiogram was performed and a giant aneurysm supplied by the splenic artery was disclosed (fig. 2). The splenic angiogram showed profuse extravasation of the contrast medium from the aneurysm into the peritoneal cavity. Arterial embolization with insertion of metallic coils into the aneurysm was undertaken and the patient was sent back to the wards with relative stable hemodynamics. His clinical course was acceptable with progressive recovery after the transarterial embolization. On the 42nd day of hospitalization a follow-up transabdominal ultrasound still showed a $6.8 \mathrm{~cm}$ pseudocyst in the tail of the pancreas, however no turbulent blood flow could be detected by color Doppler imaging this time, probably denoting a satisfactory effect of the embolization ( $\underline{\text { fig. }} 3$ ). The patient was finally dismissed in a stable condition and still follows regular visits to the outpatient clinic 2 years after the successful life-saving embolization.

\section{Discussion}

Among the complications of chronic pancreatitis, pseudoaneurysmal formations are rare with a prevalence probably $<10 \%$, but these abnormal vascular formations may carry a severe outcome with a mortality rate $>90 \%$ in untreated patients. Pancreatic pseudoaneurysm is the result of erosion of the pancreatic or peripancreatic artery into a pseudocyst, and the most commonly involved arteries in order of frequency are the splenic artery with up to $50 \%$ of cases, the gastroduodenal artery in $15 \%$, and less frequently the pancreaticoduodenal artery, the superior mesenteric artery, the left gastric artery and the hepatic artery [1-3]. The wall of the pseudoaneurysm is composed of fibrous tissue instead of a well-formed artery as seen in true aneurysm, and one of the distinct characteristics is its rapid enlargement as a pulsating vascular malformation; it may bleed into the biliary tree, pancreatic duct ('hemosuccus pancreaticus'), gastrointestinal tract, peritoneal cavity and retroperitoneum. The hemorrhage is usually associated with a very poor prognosis $[4,5]$. Transabdominal ultrasound with Doppler and contrast-enhanced computed tomography are useful noninvasive diagnostic methods in critically ill patients, but usually angiography is required to confirm the diagnosis. In our case, the use of color Doppler with color flow imaging and pulsed Doppler spectral analysis established the diagnosis of pancreatic pseudoaneurysm while contrast-enhanced computed tomography clearly defined the active bleeding. Various treatment modalities are proposed in the management of pancreatic pseudoaneurysm such as transarterial catheter embolization [6-9], percutaneous injection of thrombin $[10,11]$ and surgical intervention [12-14].

Some authors still advocate that transarterial catheter embolization should only be a palliative approach before surgical intervention such as primary pancreatic resection is feasible and is not indicated in emergent conditions for patients with hemodynamic 
instability and hemorrhagic shock [15]. There is a consensus that all these vascular malformations should be treated, however it is still controversial whether transarterial catheter embolization itself may be the definite management or whether it should be only an intermediate step before surgery. We herein report a very rare case of pancreatic pseudoaneurysm rupture with hemodynamic shock which responded successfully to arterial embolization with no need for surgery.

\section{Disclosure Statement}

No conflicts of interests are declared by the authors.

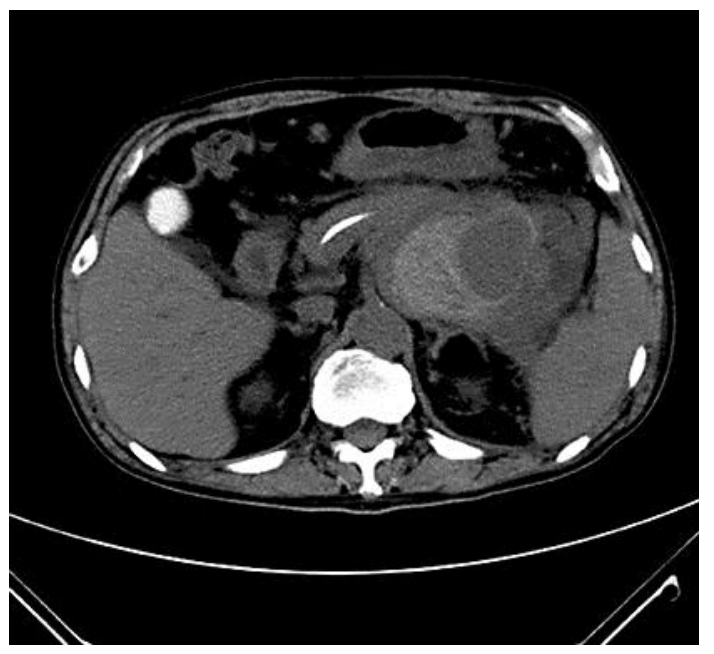

Fig. 1. Contrast-enhanced CT during the arterial phase showed a large hyperdense structure in the tail of the pancreas with extensive enhanced peripheral areas. 


\begin{tabular}{r|l|l|l} 
Case Reports in & $\begin{array}{l}\text { Case Rep Gastroenterol 2012;6:254-259 } \\
\text { DOI: } 10.1159 / 000338845\end{array}$ & $\begin{array}{l}\text { Published online: } \\
\text { May 8, 2012 }\end{array}$ & $\begin{array}{l}\text { @ 2012 S. Karger AG, Basel } \\
\text { ISSN 1662-0631 } \\
\text { www.karger.com/crg }\end{array}$ \\
\hline
\end{tabular}

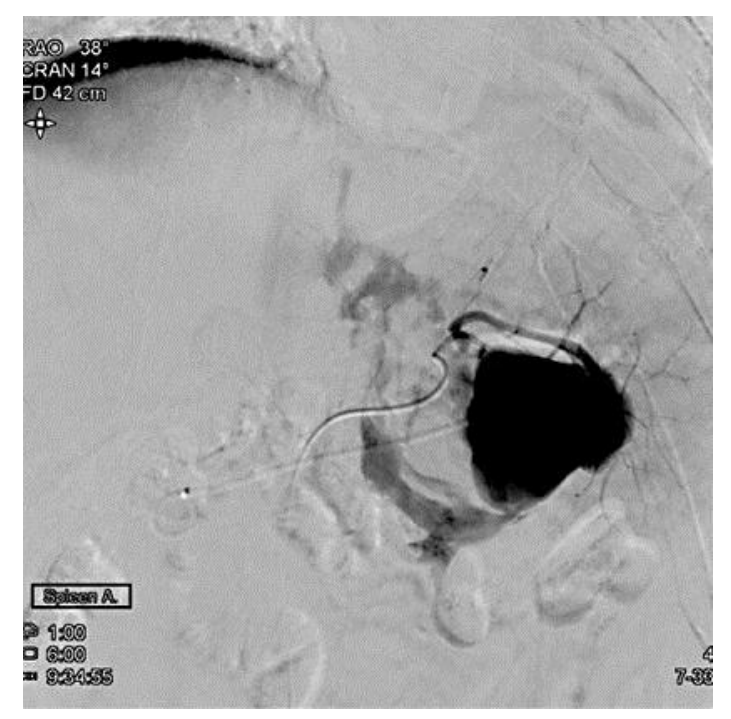

Fig. 2. A large vascular malformation with brisk contrast extravasation during angiography.

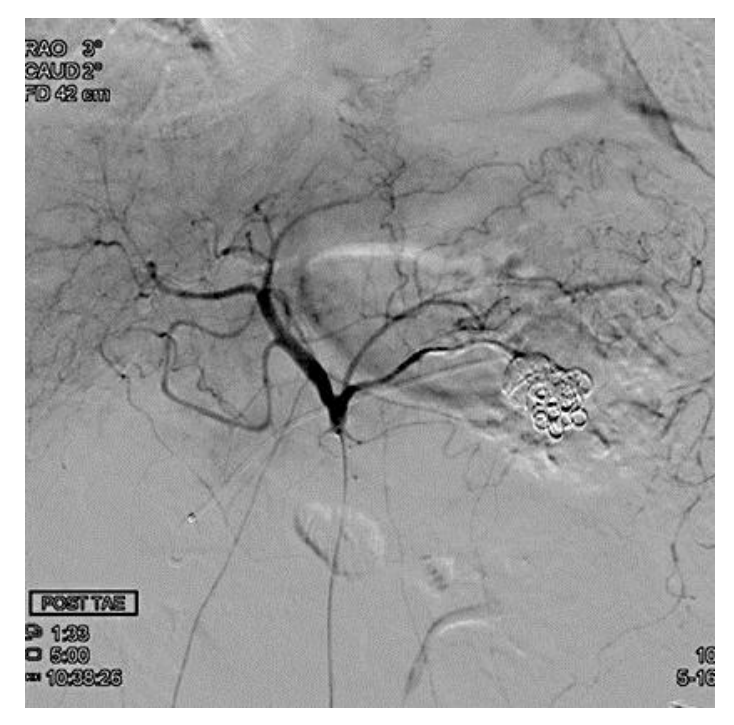

Fig. 3. Extravasation of the contrast medium was completely arrested after arterial embolization.

\section{References}

1 El Hamel A, Parc R, Adda G, Bouteloup PY, Huguet C, Malafosse M: Bleeding pseudocysts and pseudoaneurysms in chronic pancreatitis. Br J Surg 1991;78:1059-1063.

2 Carr JA, Cho JS, Shepard AD, Nypaver TJ, Reddy DJ: Visceral pseudoaneurysms due to pancreatic pseudocysts: rare but lethal complications of pancreatitis. J Vasc Surg 2000;32:722-730.

-3 Ammori BJ, Madan M, Alexander DJ: Haemorrhagic complications of pancreatitis: presentation, diagnosis and management. Ann R Coll Surg Engl 1998;80:316-325.

4 Hsu JT, Yeh CN, Hung CF, Chen HM, Hwang TL, Jan YY, Chen MF: Management and outcome of bleeding pseudoaneurysm associated with chronic pancreatitis. BMC Gastroenterol 2006;11:3. 
5 Tessier DJ, Stone WM, Fowl RJ, Abbas MA, Andrews JC, Bower TC, Gloviczki P: Clinical features and management of splenic artery pseudoaneurysm: case series and cumulative review of literature. J Vasc Surg 2003;38:969-974.

-6 Udd M, Leppäniemi AK, Bidel S, Keto P, Roth WD, Haapiainen RK: Treatment of bleeding pseudoaneurysms in patients with chronic pancreatitis. World J Surg 2007;31:504-510

7 Stösslein F, Zimmermann L, Bulang T: Embolization treatment of bleeding complications in pancreatitis. J Hepatobiliary Pancreat Surg 1998;5:344-347.

-8 Mandel SR, Jaques PF, Sanofsky S, Mauro MA: Nonoperative management of peripancreatic arterial aneurysms. A 10-year experience. Ann Surg 1987;205:126-128.

-9 Gambiez LP, Ernst OJ, Merlier OA, Porte HL, Chambon JP, Quandalle PA: Arterial embolization for bleeding pseudocysts complicating chronic pancreatitis. Arch Surg 1997;132:1016-1021.

10 Krueger K, Zaehringer M, Lackner K: Percutaneous treatment of a splenic artery pseudoaneurysm by thrombin injection. J Vasc Interv Radiol 2005;16:1023-1025.

11 Luchs SG, Antonacci VP, Reid SK, Pagan-Marin H: Vascular and interventional case of the day. Pancreatic head pseudoaneurysm treated with percutaneous thrombin injection. AJR Am J Roentgenol 1999;173: 830-834.

12 Bender JS, Bouwman DL, Levison MA, Weaver DW: Pseudocysts and pseudoaneurysms: surgical strategy. Pancreas 1995;10:143-147.

-13 Stabile BE, Wilson SE, Debas HT: Reduced mortality from bleeding pseudocysts and pseudoaneurysms caused by pancreatitis. Arch Surg 1983;118:45-51.

-14 Bresler L, Boissel P, Grosdidier J: Major hemorrhage from pseudocysts and pseudoaneurysms caused by chronic pancreatitis: surgical therapy. World J Surg 1991;15:649-652.

15 Woods MS, Traverso LW, Kozarek RA, Brandabur J, Hauptmann E: Successful treatment of bleeding pseudoaneurysms of chronic pancreatitis. Pancreas 1995;10:22-30. 